\title{
Price-based load coordination revisited: augmenting open-loop coordination approaches
}

\author{
Christoph M. Flath ${ }^{1}$ - Sebastian Gottwalt ${ }^{2}$
}

Received: 2 February 2015/Accepted: 15 February 2016/Published online: 29 February 2016

(C) The Author(s) 2016. This article is published with open access at Springerlink.com

\begin{abstract}
The activation of flexible loads through demand side management offers opportunities for more efficient power systems operations. Price-based incentive are a straight-forward form for decentral coordination of these flexible loads. However, their applicability has recently been seen more pessimistic as they may induce new load peaks due to herding effects. We revisit these results by characterizing desynchronized posted pricing approaches. Illustrating highly flexible load by means of electric vehicle charging, we show that these desynchronized rate can mitigate the occurrence of extreme load spikes, improve the utilization of renewable generation and in summary create significant system cost savings. Our results show that simple open-loop pricing can almost match the efficiency of closed-loop adaptive pricing in settings with limited system flexibility. We find that the more renewable generation and flexible load are present in the system, the better more complex pricing schemes fare compared to simple ones. This insight may guide regulators and utilities in establishing more effective pricing schemes in retail electricity markets.
\end{abstract}

Keywords Smart grid · Electricity pricing - Demand side management . Electric vehicles $\cdot$ Renewable energy sources

Sebastian Gottwalt

gottwalt@fzi.de

Christoph M. Flath

christoph.flath@uni-wuerzburg.de

1 University of Würzburg, Josef-Stangl-Platz 2, 97070 Würzburg, Germany

2 FZI Research Center for Information Technology, Haid-und-Neu-Str. 10-14, 76131 Karlsruhe, Germany 


\section{Introduction}

An efficient integration of volatile renewable energy sources into the power grid calls for a more flexible demand side to minimize the need for expensive balancing power and storage capacity. Smart grids enhance the existing grid infrastructure through the provision of bi-directional information and communication technology (Blumsack and Fernandez 2012). Using dynamic electricity rates, utilities can tap into demand side flexibility (Faruqui et al. 2010). However, recent research has been more pessimistic with respect to the coordination capabilities of price signals due to the tendency of creating herding or load synchronization effects (Gottwalt et al. 2011; Sioshansi 2012). Ramchurn et al. (2012) note that [Real-time pricing] "can create unexpected peaks in demand, when all individuals respond to a signal in the same way, and inadvertently synchronize with others". They conclude that "demand-side management technologies that simply rely on reacting to control or price signals will not be enough". This calls for adaptive customer prices dynamically reflecting current grid conditions in the spirit of optimal spot pricing (Schweppe et al. 1988). At the same time, Dütschke and Paetz (2013) point out that customer acceptance will require simple and reliable pricing schemes.

Can this obvious disparity between customer preferences (simple and reliable price signals) and system requirements (effective load coordination) be softened? To facilitate the integration of ever higher levels of renewable generation, finding a solution to this challenge is of great interest to utilities and regulators around the globe. This paper revisits this price-coordination conundrum and explores rate design options to reduce synchronization under exogenously specified electricity rates in different power system configurations. Specifically, we want to address the following research questions:

- What are suitable desynchronization approaches for posted price schemes and to what extent do they limit herding effects?

- What is the effect of different price regimes on system costs under varying renewable generation capacities and flexible load penetration?

- To what extent can simple posted pricing schemes achieve satisfying coordination results and when do they fail?

The remainder of this paper is structured as follows: Sect. 2 provides an overview of related research. To analyze the impacts on the aggregate power system, Sect. 3 introduces a local microgrid model featuring renewable wind and conventional generation as well as flexible and inflexible loads. Using different pricing schemes, we then analyze the aggregate charging load of an EV fleet in Sect. 4. Here, we start with standard rate designs (flat, real-time, time-of-use) and subsequently introduce additional design elements (power surcharges, randomized group rates) to reduce load clustering effects. In Sect. 5 we analyze the system costs of different rates in different configurations of a local microgrid and we carry out a sensitivity analysis for randomized group rates to identify important parameters for rate design. Finally, Sect. 6 concludes and presents an outlook for future research opportunities. 


\section{Related work}

Demand side management (DSM), i.e., the active coordination of load flexibility, can offer sizeable control potentials at much lower costs than the expansion of storage capacities (Strbac 2008). Similarly, Ramchurn et al. (2012) argue that one main objective of a future Smart Grid is the integration of fluctuating renewable generation through flexible loads. Albadi and El-Saadany (2008) categorize DSM programs as incentive- and price-based regimes. Under incentive-based programs customers cede load control to the system operator or an intermediary (e.g., an energy retailer or demand response aggregator) in exchange for more favorable contract conditions (e.g., lower base fee). This corresponds to a setting of centralized load control. Price-based programs emphasize a decentralized decision paradigm by applying dynamic pricing to incentivize changes of customer behavior (Borenstein et al. 2002).

\subsection{Centralized load control}

In centralized control schemes, a designated entity, typically referred to as "aggregator" or "load controller", schedules the operation of flexible loads (Subramanian et al. 2013). Under full information, a central operator will clearly be able to determine an optimal (i.e., minimal costs or emissions) dispatch schedule for the loads. At the same time, standard drawbacks of centralized regimes apply, e.g., security and privacy concerns, computational complexity of large-scale optimization, or incentive compatibility problems may arise. To mitigate some of these problems, several authors propose hierarchical schemes where load subgroups are controlled by a local aggregator, e.g., on the distribution grid level (Callaway and Hiskens 2011).

\subsection{Decentralized load control}

Lamparter et al. (2010) note, that in smart grids "a central fully informed entity is not available due to natural information asymmetries and selfish participants (suppliers/consumers)". Therefore, central approaches are either highly inefficient or not applicable, especially in the case of direct control of electrical appliances in private households. This observation necessitates decentralized decision regimes which have lower information and computation requirements and can fully retain customer incentives and privacy concerns (Vandael et al. 2011). Due to the distributed nature of this control paradigm, a large scale application requires a careful analysis of the emergent system behavior (Ramchurn et al. 2011). Openloop and closed-loop are two basic principles for decentralized load control. Figure 1 sketches decision making schemes for these two principles. 


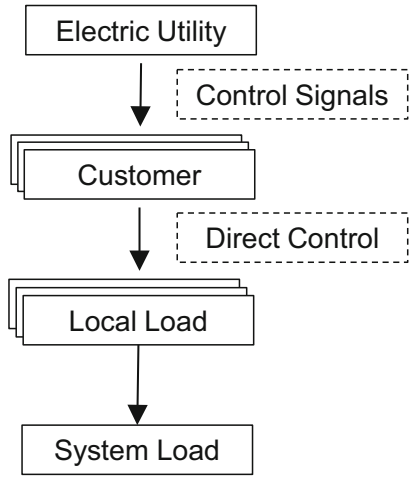

Open-loop control

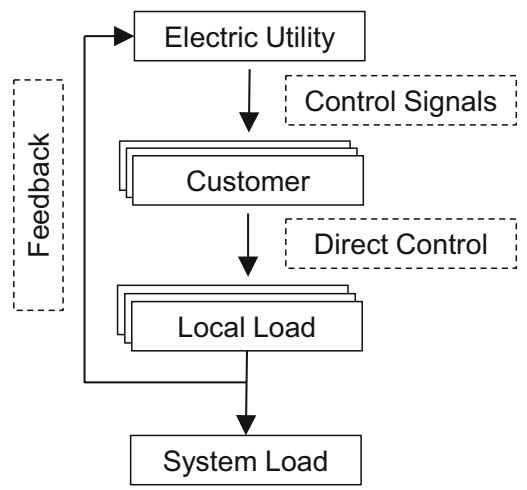

Closed-loop control

Fig. 1 Open-loop and closed-loop decision making schemes for decentral coordination

\subsubsection{Open-loop control}

Most commonly, open-loop control in residential DSM is instantiated using pricebased coordination. This will require some form of dynamic pricing, e.g., time-ofuse (TOU) pricing, critical peak pricing (CPP), or real-time pricing (RT). Electric utilities carried out various dynamic pricing studies showing that customers are responsive to changes in electricity prices (Faruqui and Palmer 2012). However, recent research has been more pessimistic with respect to the coordination capabilities of price signals due to the tendency of creating herding or load synchronization effects (Gottwalt et al. 2011). Ramchurn et al. (2012) note that real-time pricing "can create unexpected peaks in demand, when all individuals respond to a signal in the same way, and inadvertently synchronize with others". They conclude that "demand-side management technologies that simply rely on reacting to control or price signals will not be enough".

\subsubsection{Closed-loop control}

More sophisticated coordination approaches address this problem. Mohsenian-Rad et al. (2010) analytically show that such an approach will yield an efficient allocation in a general setting. Using learning agents, Ramchurn et al. (2011) demonstrate that a feedback loop in a RT pricing regime will achieve efficient decentral coordination. Gan et al. (2013) propose an iterative charging control for electric vehicles (EV) for valley filling. Based on tentative charging decisions of the EVs the utility adapts electricity prices. While closed-loop coordination approaches may in theory guarantee almost optimal results, a real-world application for retail customers is difficult to implement as it requires them to actively bid on spot markets and thus expose them to quantity and price risk as clearing prices and amounts are stochastic (Bitar and Low 2012). Consequently, in practice RT pricing 
schemes are often posted price schemes where customers receive a fluctuating yet reliable price signal.

\subsection{Customer acceptance and adoption}

Both Goett et al. (2000) and Dütschke and Paetz (2013) find that customer acceptance is decreasing in pricing complexity and dynamics. Customer acceptance is key to achieve wide-range adoption of demand response. Woo et al. (2008) summarize this issue as follows: "If consumers do not understand a tariff, they cannot respond to the tariff's price signals. The lack of understanding also contributes to customer rejection of the tariff's mandatory implementation". Similarly, Homburg et al. (2014) find that customers often prefer simple over less expensive yet more complex prices. Thus both suppliers as well as regulators should focus on establishing simple electricity pricing schemes. Besides the extrinsic adoption motive price, there are intrinsic adoption factors such as environmentalism, the utility and usability of the end user devices, the age, or income (Wunderlich et al. 2013a, b). While these are abstracted from in the remainder of this paper, they also need to be considered when designing electricity rates. Ideally, suppliers should strive to establish demand response service offerings that convince customers along all relevant dimensions-including pricing simplicity.

\subsection{Randomized load control}

Acknowledging the overcoordination problems of price-based load control and the complexity of market-based allocation schemes, recent research contributions have put forward the "power of randomness". The travel and hospitality industry applies randomization successfully in practice in the form of "opaque selling" (Jiang 2007; Fay 2008). Here, customers are offered a generic product (e.g., "4-star-hotel in Rome') through an intermediary with the concrete realization (e.g., Hilton vs. Marriot vs. Intercontinental) not being disclosed at the time of the sale.

In the smart grid context, Shinwari et al. (2012) propose transmitting operation probabilities for shiftable loads to local control agents. The probabilities for starting shiftable loads are high in hours with low non-shiftable load in the system. Van Den Briel et al. (2013) extend this approach by determining operation probabilities based on the non-shiftable loads. Kishore and Snyder (2010) apply a stochastic admission control scheme from the telecommunications sector to avoid simultaneous load occurrences. In a similar fashion, Gong et al. (2012) demonstrate that randomized charging for electric vehicles can reduce transformer wear. While these approaches characterize ways to break the problem of price-induced herding of loads, they do not characterize appropriate incentive structures that induce truthful behavior of system participants.

Our analysis revisits price-based coordination of flexible loads in smart grids. We highlight load synchronization problems under standard electricity pricing approaches (RTP, TOU). Subsequently, we introduce rate modifications to improve the coordination performance. 


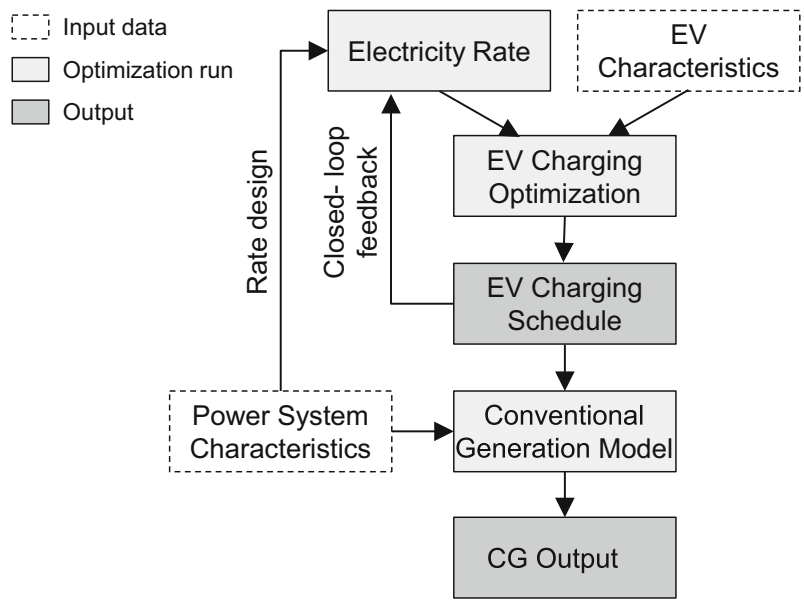

Fig. 2 Overview of simulation flow

\section{Microgrid model}

To analyze the emergent behavior of flexible loads under different rate scenarios, we model a microgrid with local generation and load. We consider different generation options as well as inflexible base load and flexible EV charging loads. In the following, we provide a model overview and then characterize the underlying models of the demand and supply side.

\subsection{Overview}

Our microgrid model consists of three sequential control problems-rate design, flexible load response and generation dispatch (Fig. 2). First, the supplier determines a rate and transmits it to the customer pool. The supplier will try to signal wind availability and base load characteristics to flexible loads by means of this price signal. The customers will then internalize this price information and correspondingly schedule their (flexible) EV loads. In the special case of closed-loop adaptive prices the rate design and load response steps are performed simultaneously. Finally, given the realized demand choices, the supplier plans conventional generation output in a cost-minimizing manner to serve the residual load which remains after accounting for renewable generation.

\subsection{Demand side}

\subsubsection{Inflexible load}

The base load is constituted by 900 households which are modeled using the seasonally adjusted H0 household load profile from the German utility association. ${ }^{1}$

\footnotetext{
${ }^{1}$ http://www.bdew.de/internet.nsf/id/DE_Standartlastprofile.
} 
These load profiles are reported in 15 min intervals which we adopt as the size of a time slot in our optimization model. We assume static behavior as well as a constant number of these loads across all scenarios. We refer to total base load at time $t$ as $L_{t}^{B}$.

\subsubsection{Flexible load}

In general, integration of flexible loads can be characterized as a cost minimization problem with appropriate constraints reflecting flexibility endowment and technical characteristics (Petersen et al. 2013). Depending on the underlying electricity consumer, load flexibility can come along in very different flavors, e.g., temporal flexibility (load shifting), different intensity levels (load curtailment) or interruption (load shedding). To explore the potentials of price-based load coordination, it is instrumental to consider a load type with very high flexibility. Electric vehicle charging gives rise to large and flexible loads with respect to both power level and time. Hence, they are a prime candidate for exploring the effects of load flexibility (Blumsack and Fernandez 2012). Following previous research (e.g., Sioshansi 2012; Flath et al. 2014), we model an EV fleet by combining empirical driving profiles and appropriate EV technical specifications with an individual charging decision model. To this end, we extracted 900 profiles from the German Mobility Panel. ${ }^{2}$ The driving profiles are sampled in 15 min intervals and specify driving distance and purpose. We assume near-future EV specifications with battery size $\bar{B}=30 \mathrm{kWh}$, energy consumption of $0.15 \mathrm{kWh}$ per $\mathrm{km}$ and maximum charging power $\bar{P}=11$ $\mathrm{kW}$. Energy usage from driving $d_{t}$ obtains from the distance driven and the energy consumption rate. The battery level at time $t$ is given by $\beta_{t}$. We use greek letters to indicate decision variables. Charging is assumed to be possible at home and at the workplace. The charging availability at time $t$ is coded as a binary parameter $a_{t}$. We consider different penetration levels 33, 66 or $100 \%$ of the 900 households own an electric vehicle.

Our charging model accounts for both cost-oriented as well as mobility-oriented charging motives and encapsulates both the polar benchmarks of simple (as fast as possible) and optimal smart charging as established in prior research (Lopes et al. 2010; Schuller et al. 2014). Optimal smart charging is characterized as a linear charging cost minimization program over time horizon where charging decisions are constrained by technical (battery level, charging speed, vehicle location) as well as usage (driving requests need to be served) requirements. We adapt this approach to not only reflect cost minimization but also to account for vehicle availability levels in-tune with the range anxiety phenomenon (Franke et al. 2012). To this end, we include a concave benefit term $\omega=R \sqrt{\bar{\beta}},{ }^{3}$ where $\bar{\beta}=\frac{1}{\bar{B} \cdot T} \sum_{t \in[1 . . T]} \beta_{t}$ is the average battery level over the optimization horizon which captures the vehicle availability and $R$ is a driver-specific weighting parameter measuring a driver's range anxiety. The function's concavity ensures decreasing marginal value of EV availability

\footnotetext{
2 http://daten.clearingstelle-verkehr.de/192/.

${ }^{3}$ The square root relationship is the only concave function applicable in a quadratic programming setting. It is modeled by constraining $\omega^{2}$.
} 
which resonates well with standard behavioral assumptions. The weight term allows us to model heterogeneous charging behavior ranging from flexible, cost-oriented (low $R$ ) to impatient, range-focused (high $R$ ) charging behavior. We draw parameter realizations from an exponential distribution. Consequently, the modeled EVs are not only differing in their driving profiles but also in their charging behavior. This helps to minimize simulation artifacts which are due to homogeneous charging behavior. Dynamic electricity rates are given by $p_{t}$ and decisions on EV charging amounts are denoted $\phi_{t}$. We then obtain the electric vehicle optimization program:

$$
\min \left(\sum_{t \in[1 . . T]} p_{t} \cdot \phi_{t}-R \omega\right)
$$

subject to:

$$
\begin{gathered}
\beta_{t}=\beta_{t-1}+\phi_{t}-d_{t} \quad \forall t \in[T] \equiv\{1, \ldots, T\} \\
0 \leq \beta_{t} \leq \bar{B} \quad \forall t \in[T] \\
\beta_{0} \leq \beta_{T} \\
\omega^{2} \leq \bar{\beta} \quad \forall t \in[T] \\
0 \leq \phi_{t} \leq a_{t} \cdot \bar{P} \quad \forall t \in[T]
\end{gathered}
$$

Constraint (2) includes charging amounts $\left(\phi_{t}\right)$ and driving energy requirements $\left(d_{t}\right)$ to ensure continuity of the battery level $\left(\beta_{t}\right)$. To avoid complete discharging at the end of the weekly optimization horizon, we require the terminal battery level $\beta_{T}$ to at least match the initial value $\beta_{0}$ (Constraint 4 ). Note that smart charging will never forfeit trips and thus guarantees to meet the driving needs of the user. Other constraints like minimum spontaneous range or avoidance of high loads can be easily integrated in this model. ${ }^{4}$ In the absence of incentives (i.e., a flat electricity tariff), load flexibility cannot meaningfully be tapped into in the sense of a cost minimization problem (electricity price is constant over time). Consequently, EV charging optimization is equivalent to maximizing the $\omega$ term. In this case, drivers will charge the required energy amounts as early as possible and in turn maximize their average battery SOC. The aggregate charging load of the whole EV fleet at time $t$ is denoted $L_{t}^{F}$.

\subsection{Supply side}

To serve the flexible charging and the static household loads, the utility needs to optimally procure gas turbine (GT) capacity, dispatch this capacity and decide on transactions on the reserve market while at the same time integrating available (zero-cost) wind generation. To this end, flexible loads should ideally be scheduled such that the residual load, which needs to be covered through costly conventional

\footnotetext{
${ }^{4}$ See Qian et al. (2011) or Sioshansi et al. (2010) for alternative model formulations.
} 
generation, is minimized. Furthermore, scheduling needs to establish a generation plan which ensures efficient operation of the conventional generators.

\subsubsection{Renewable generation}

To model renewable generation output, we leverage empirical wind generation data retrieved from the $50 \mathrm{~Hz}$ balancing zone in Germany. ${ }^{5}$ We convert this raw data to a dimensionless "wind yield curves" with support over $[0,1]$ which describes the fluctuations of the relative wind output level over time. We then combine this yield curve with three different wind generation capacity scenarios to explore different penetration levels of RES production per time slot $q_{t}^{\mathrm{RES}}$. We choose installed capacity to match 100,200 or $300 \%$ of the maximum base load level over the simulation horizon. We refer to these settings as "low", "mid" and "high" wind scenario.

\subsubsection{Conventional generation}

The dispatch problem for the microgrid's conventional generators is formulated as a quadratic, mixed-integer program minimizing total generation costs comprising of gas turbine fuel costs as well as the costs of procuring from the reserve market. The problem is subject to constraints reflecting gas turbine capacity $K$ and operational characteristics as well as the power system. In the evaluation, $K$ is specified scenario-specific depending on the number of EVs and the renewable generation capacity (see Table 1). ${ }^{6}$ Besides the turbine output decisions $\theta_{t}^{\mathrm{GT}}$, the supplier also needs to manage the turbine's operational state $\psi_{t}$ by means of start up decisions $\zeta_{t}$.

The cost components are modeled as follows: Gas turbine fuel costs are governed by turbine output, turbine efficiency and the natural gas price. Current market prices are approximately $3 € / \mathrm{MMBtu}$ which translates to $0.0102 € / \mathrm{kWh}$. Turbine efficiency is increasing in the utilization level $\theta_{t}^{\mathrm{GT}} / K$ : at the minimum utilization level $40 \%$ efficiency is around $47 \%$, at full load it peaks at $58.5 \%$ (Los et al. 2009). We assume a simplified linear efficiency trajectory which allows us to characterize output-dependency of $\operatorname{costs} c^{\mathrm{GT}}$ in a linear fashion, that is

$$
c^{\mathrm{GT}}\left(\theta_{t}^{\mathrm{GT}}\right)=0.0147 \theta_{t}^{\mathrm{GT}}+\psi_{t} 0.0028 K \text {. }
$$

The reserve power costs $c^{R}$ are modeled as a quadratic function of reserve energy quantity $\theta_{t}^{R}$ to capture increasing marginal costs of generation. We obtained function parameters from a quadratic fit of the peak electricity price model proposed by Grünewald et al. (2014):

\footnotetext{
5 We use data from the year 2013 which can be accessed at http://www.50hertz.com/de/167.htm.

${ }^{6}$ For each scenario we determine the optimal capacity under flat pricing and use this for all pricing regimes.
} 


$$
c^{R}\left(\theta_{t}^{R}\right)=2.22 \times 10^{-2} \theta_{t}^{R}+6.8 \times 10^{-5}\left(\theta_{t}^{R}\right)^{2}
$$

Given these decision variables and cost components, the microgrid dispatch problem is formulated as follows:

$$
\min \sum_{t}\left(c^{\mathrm{GT}}\left(\theta_{t}^{\mathrm{GT}}\right)+c^{R}\left(\theta_{t}^{R}\right)\right)
$$

subject to:

$$
\begin{gathered}
q_{t}^{\mathrm{RES}}+\theta_{t}^{\mathrm{GT}}+\theta_{t}^{R} \geq L_{t}^{F}+L_{t}^{B} \quad \forall t \in[T] . \\
\psi_{t} \cdot 0.4 K \leq \theta_{t}^{\mathrm{GT}} \leq \psi_{t} \cdot K \quad \forall t \in[T] \\
\psi_{t+i} \geq \zeta_{t} \quad \forall i \in\{1,2, \ldots, 6,7\}, \quad \forall t \in[T] \\
\psi_{t} \leq \psi_{t-1}+\zeta_{t} \quad \forall t \in[T] \\
\psi_{t}, \zeta_{t} \in\{0,1\}
\end{gathered}
$$

Equation (10) ensures sufficient generation to cover the active load. Following Varaiya et al. (2011), we only require local generation adequacy (total generation $\geq$ load) instead of strict equality which reflects the shedding potential of renewable generators. Capacity limits and minimum output levels for the gas turbine are enforced through (11) - both contingent on the system being up and running $\left(\psi_{t}=1\right)$. A minimum turbine uptime of $2 \mathrm{~h}$ (cf. van Dijken et al. 2010) is established by Eq. (12). Finally, start decisions and turbine state changes are consistently linked in Constraint (13).

\section{Load evaluation}

We first analyze standard electricity pricing schemes to determine the key factors leading to load synchronization. Based on the insights, we explore rate modifications to reduce load synchronization. To exemplify the effects of the distinct rates we show the customer tariff and the corresponding composition of generation (RES, gas turbine and reserve) and load (household and EV charging) for week 17 in 2013. The results are based on a simulation scenario with high wind generation capacities and $100 \% \mathrm{EV}$ penetration.

\subsection{Flat electricity tariff}

The left panel of Fig. 3 depicts the aggregate charging loads of an EV fleet under a flat tariff. In absence of monetary incentives for shifting EV owner will charge as-fast-aspossible to maximize their average battery level, that is the benefit term $\omega$ in Eq. (1). We can observe that charging loads are evenly distributed and large spikes can be avoided. Under a flat tariff there is no possibility to influence EV charging activity. Hence, charging often takes place during times with no or very limited availability of 

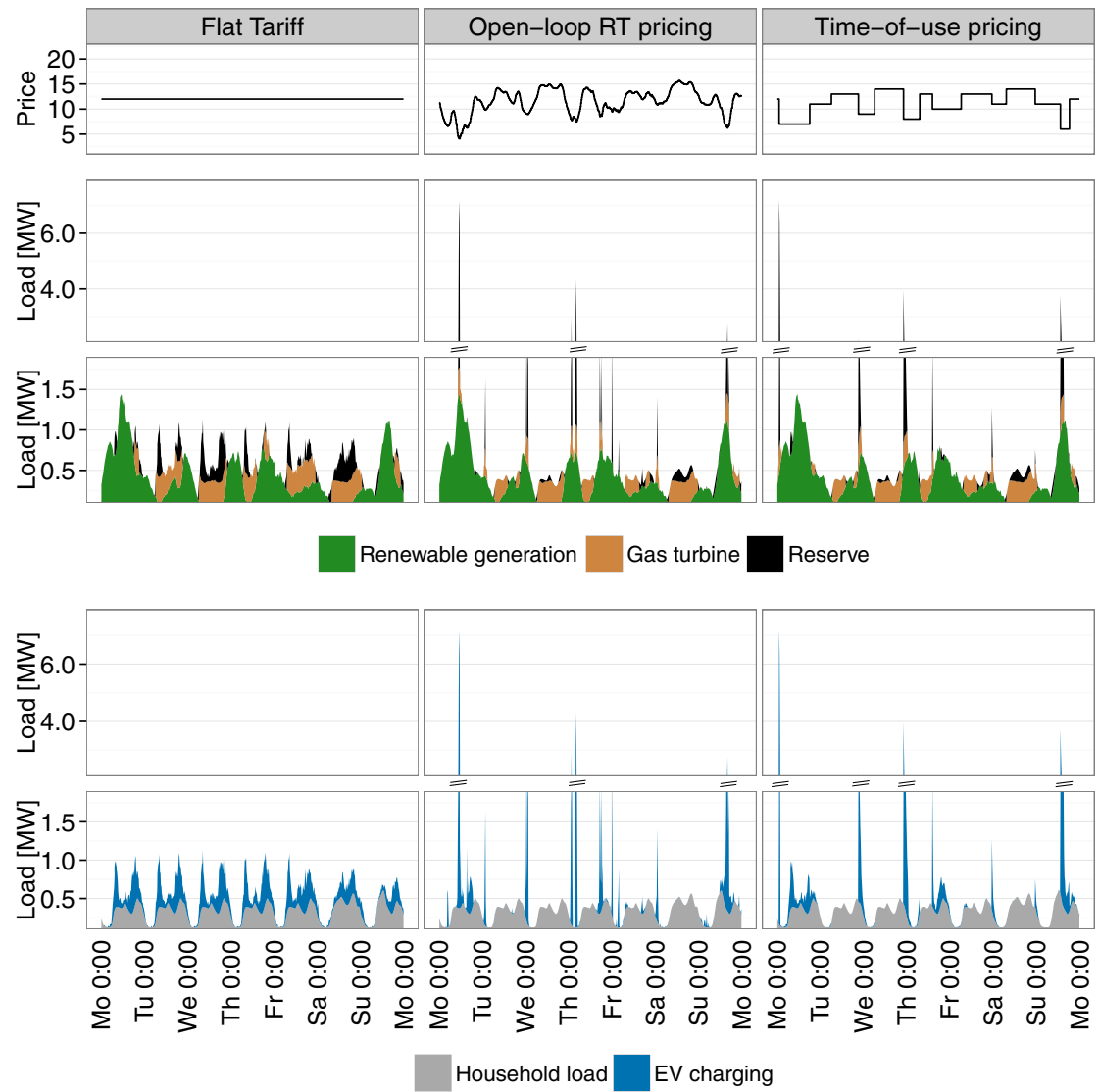

Fig. 3 Rate structure and the corresponding load and generation behavior for flat, open-loop real-time and time-of-use pricing ( $y$-axis is broken to accommodate the load spikes under open-loop RT and TOU pricing)

wind generation. Consequently, in many situations the gas turbine and reserve electricity is needed while at other times renewable generation remains unused.

\subsection{Open-loop real-time pricing}

Under open-loop RT pricing, the retail price reflects the availability of renewable generation for the flexible loads. We create open-loop real-time prices as a linear function of net renewable generation. ${ }^{7}$ To facilitate presentation, the tariffs are appropriately scaled by means of a constant. Denoting by $\overline{\mathrm{RES}}$ the largest RES availability value over the simulation horizon, we obtain

\footnotetext{
7 Results do not change qualitatively for other functional relationships. Compared to the dual values from the power system optimization problem for base load, this pricing formula can differentiate between different levels of surplus wind generation where the dual would always be zero.
} 


$$
p_{t}^{\mathrm{RT}}=\overline{\mathrm{RES}}-\left(q_{t}^{\mathrm{RES}}-L_{t}^{B}\right) .
$$

The resulting rate structure is illustrated in the center panel of Fig. 3. If EV owners base their charging decision on this ex-ante specified tariff, a high concentration of EV loads can be observed. Such herding effects are in-line with results from prior research on the effects of price-based coordination in retail electricity markets. At the same time, concentrated EV charging-in our example scenario-largely exceeds available renewable generation and requires very high amounts of reserve electricity. Therefore, load synchronization is due to the presence of distinct time slots with minimal costs to which the flexible loads will respond in a common manner.

\subsection{Time-of-use pricing}

Time-of-use prices constrain the rate structure to a limited number of rate zones and this way remove distinct minimum prices. We create time-of-use rates such that the levels and length of the price zones minimize the deviation from the RT price presented before. To this end, we apply another mixed-integer program which determines the optimal rate structure $\rho_{t}^{\text {TOU }}$ by placing a limited number of price jumps (13 jumps yield 14 rate zones) over a time interval (in our case 1 week)

$$
\min \sum_{t=1}^{T}\left|\rho_{t}^{\mathrm{TOU}}-\rho_{t}^{\mathrm{RT}}\right| .
$$

Additional decision variables $\left(\eta_{t}^{+/-}, \delta_{t}^{+/-}\right)$are used to ensure a valid TOU structure by means of the subsequent constraints where $M$ denotes the standard big-M modeling approach: ${ }^{8}$

$$
\begin{gathered}
\rho_{t}^{\mathrm{TOU}}=\rho_{t-1}^{\mathrm{TOU}}+\delta_{t}^{+}-\delta_{t}^{-} \quad \forall t \in[T] \\
\delta_{t}^{+} \leq \eta_{t}^{+} \cdot M \quad \forall t \in[T] \quad \delta_{t}^{-} \leq \eta_{t}^{-} \cdot M \quad \forall t \in[T] \quad \sum_{t=1}^{\mathrm{T}}\left(\eta_{t}^{+}+\eta_{t}^{-}\right) \leq 13
\end{gathered}
$$

In the right panel of Fig. 3 we can observe that TOU rates cannot reduce the overcoordination phenomenon compared to RT pricing regimes. EV charging load is similarly concentrated and maximum load levels cannot be reduced. However, the new load peaks arise at the beginning of intervals with low prices. This observation confirms the results by Ramchurn et al. (2011). Load clustering at the boundaries of the lowest price intervals is due to the benefit term $\omega$ accounting for EV battery levels. For the same electricity price EV owners prefer charging at the beginning of low price intervals to achieve higher SOC levels earlier and increase a vehicle's range for spontaneous trips. Considering EV availability levels can also lead to a shift of charging activity to TOU zones arising earlier. This behavior can be observed in the figure where aggregate EV charging on Sunday is lower than at Thursday evening despite a higher price level for electricity. Furthermore, under

\footnotetext{
${ }^{8}$ For a more comprehensive discussion of this model we refer to Flath (2013).
} 
TOU rates EV charging often exceeds available renewable generation and conventional generation requirements remain at a high level.

\subsection{Power-based surcharge}

Under the basic rate structures, load synchronization leads to peak load occurrences and at the same time available RES generation often remains unused. A key reason for load clustering under TOU pricing is due to customers preferring earlier service over later service. This leads to a bang-bang structure of charging decisions (i.e., charge at full speed or do not charge) under energy-only pricing. To obtain intermediate charging levels and thus incentivize more spread-out charging behavior, a power-based price surcharge can be introduced. On behalf of customers, this transforms the charging optimization problem (1) to a quadratic program:

$$
\min \left(\sum_{t \in[1 . . T]} p_{t} \cdot \phi_{t}+\phi_{t}^{2}\right)-R \omega
$$

The left panel of Fig. 4 depicts the EV charging load under TOU pricing with a power-based surcharge (TOU-P). Here, higher charging power levels are more costly. While customers still aim to consume in low cost TOU zones they now distribute their charging demand more evenly. The power-surcharge mitigates peaks at the boundaries of the low price intervals. This way, load spikes can be reduced compared to standard TOU pricing. With respect to generation, the power-surcharge greatly reduces the need for reserve electricity and improves renewable generation usage as excess charging during high wind hours is reduced. Yet, system load is still primarily concentrated in the four minimum price intervals. The absolute distance of the price levels impedes shifting into other potentially desirable times for EV charging. Furthermore, a power-based surcharge entails some limitations for realworld application: Many electric appliances must run in one continuous stretch and cannot adapt their power consumption as they feature fixed load profiles. Yet, they still exhibit large temporal flexibility with respect to start time selection (Gottwalt et al. 2011). For these loads, the introduction of power-based surcharges cannot mitigate load clustering. Furthermore, the power-based surcharges necessitate greater and hence more costly metering capabilities. Finally, load-based surcharges can induce inefficiencies as they penalize individual consumption increases in uncongested situations in an unwarranted fashion (Bohn 1982).

\subsection{Randomized group pricing}

Individualization of electricity is another possibility to reduce load synchronization. To this end, Muratori and Rizzoni (2015) put forward the idea of "multi-TOU" where distinct tariff signals are distributed to a limited number of consumer pools. We extend this approach in the form of group pricing (GR) where customers are assigned to groups and members of one group receive the same electricity rate. This approach can be generalized to allow various differentiation degrees, including 

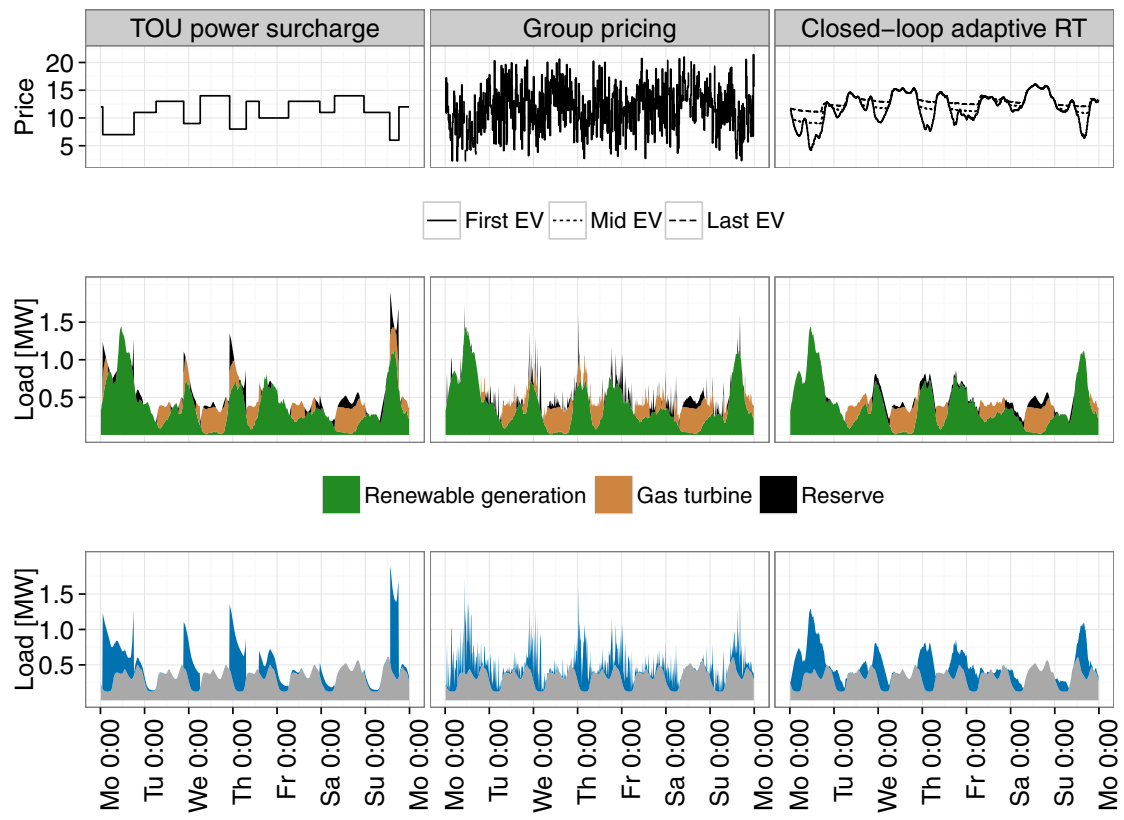

Household load EV charging

Fig. 4 Rate structure and the corresponding load and generation behavior for load desynchronization approaches

personal pricing. For an electric utility the application of group pricing requires three basic decisions - determine the number of different groups, assign customers to groups and specify a rate for each group. In the following section we analyze various group sizes including the limit case of fully individualized electricity rates. With respect to customer assignment, we only apply randomly allocation of customers to the groups. ${ }^{9}$ To individualize group rates, we adopt a randomized approach. To this end, we add noise $\epsilon_{t}(\sigma)$ to an underlying RT rate, yielding the randomized group prices $\left(p_{t}^{\text {GROUP }}\right) .{ }^{10}$ The standard deviation $\sigma$ reflects the level of rate individualization, that is how different the group-specific price vectors should be. The truncation on $\left[-p_{t}, \infty[\right.$ avoids negative retail electricity prices. The center panel of Fig. 4 depicts the aggregated EV load for 25 groups and a standard deviation level for tariff randomization, $\sigma=4$. Looking at aggregate $\mathrm{EV}$ charging load, we observe that total load is distributed and RES generation can be exploited. Therefore, group pricing can also reduce over-coordination and at the same time induces load-shifting to hours with net availability of wind power. In Sect. 5.3 we

\footnotetext{
9 Alternative group formation methods (e.g., best fit on total load) do not lead to substantially different results.

${ }^{10}$ We use a truncated normal with support $\left[-p_{t}, \infty\right]: \varphi^{T}\left(0, \sigma,-p_{t}, \infty ; x\right)=\left(\frac{\varphi\left(0, \sigma^{2} ; x\right)}{\Phi\left(0, \sigma^{2}\right)-\Phi\left(0, \sigma^{2} ;-p_{t}\right)}\right) \cdot \mathbf{1}$ $\left(x>-p_{t}\right)$.
} 
discuss in more detail the effects of randomization and group size on load synchronization and RES utilization.

\subsection{Closed-loop adaptive real-time prices}

In contrast to the above described open-loop price regimes, closed-loop price signals are adapted in response to customer actions (Mohsenian-Rad et al. 2010). To avoid the necessity for individual elasticity levels, we assume that EVs sequentially take their charging decisions for 1 week. The net wind generation is the base for determining a customer's rate offering and is adapted after each charging decision. The right panel of Fig. 4 depicts prices faced by different customers. The first EV is faced with the basic open-loop RT price. Due to lower net wind availability prices for subsequent customers increase during the times where previous charging decisions were scheduled. Consequently, customers acting later will face rate structures where the price valleys have been filled. Under closed-loop real-time pricing, aggregate load follows available wind generation perfectly and undesired load concentrations are avoided. Reserve generation is only required in hard cases, e.g., when inflexible base load exceeds available renewable generation. While adaptive prices facilitate almost optimal load coordination, they burden customers with unreliable price signals and introduce significant complexity of market communication for billing and transaction verification.

\section{Microgrid-level evaluation}

To assess the effect of different coordination approaches on the system level, we simulate twelve weeks for different microgrid configurations with varying EV penetration and renewable generation capacity scenarios. In the following, we present the results concerning conventional generation usage and realized system costs. Furthermore, we explore design options for randomized group pricing.

\subsection{Conventional generation usage}

Table 1 summarizes average utilization of the gas turbine as well as the weekly average reserve usage and the maximum reserve requirement over the simulation horizon. Results for conventional generation are in line with the observations described before. Closed-loop adaptive pricing mitigates load synchronization. This regime requires the lowest peak reserve and total reserve amount and at the same time achieves the highest gas turbine utilization levels. On the other hand, openloop real-time and time-of-use prices lead to significant over-coordination. Compared with these standard dynamic pricing approaches, TOU with power surcharge and group pricing successfully increase gas turbine utilization and reduce reserve usage (both average and peak). In the example week we observed spread-out EV charging with the lowest synchronization level for uncoordinated charging. As a result low reserve peaks can be observed in the table. Yet, total reserve requirements are high as charging only matches intermittent RES generation by coincidence and 
Table 1 Gas turbine utilization and capacity and reserve requirements for different control and power system scenarios



it often occurs during hours with low renewable generation. A flat tariff avoids load synchronization, but at the same time unused flexibility potentials deteriorate system efficiency.

Open-loop real-time and time-of-use schemes have very low gas turbine utilization and lead to excessive reserve needs. The proposed desynchronization approaches achieve high gas turbine utilization and at the same time greatly reduce reserve requirements. For low penetration levels of flexible loads the basic rates show only moderate peak reserve increases. Desynchronization approaches become more important with an increasing number of flexible loads where herding can arise. 


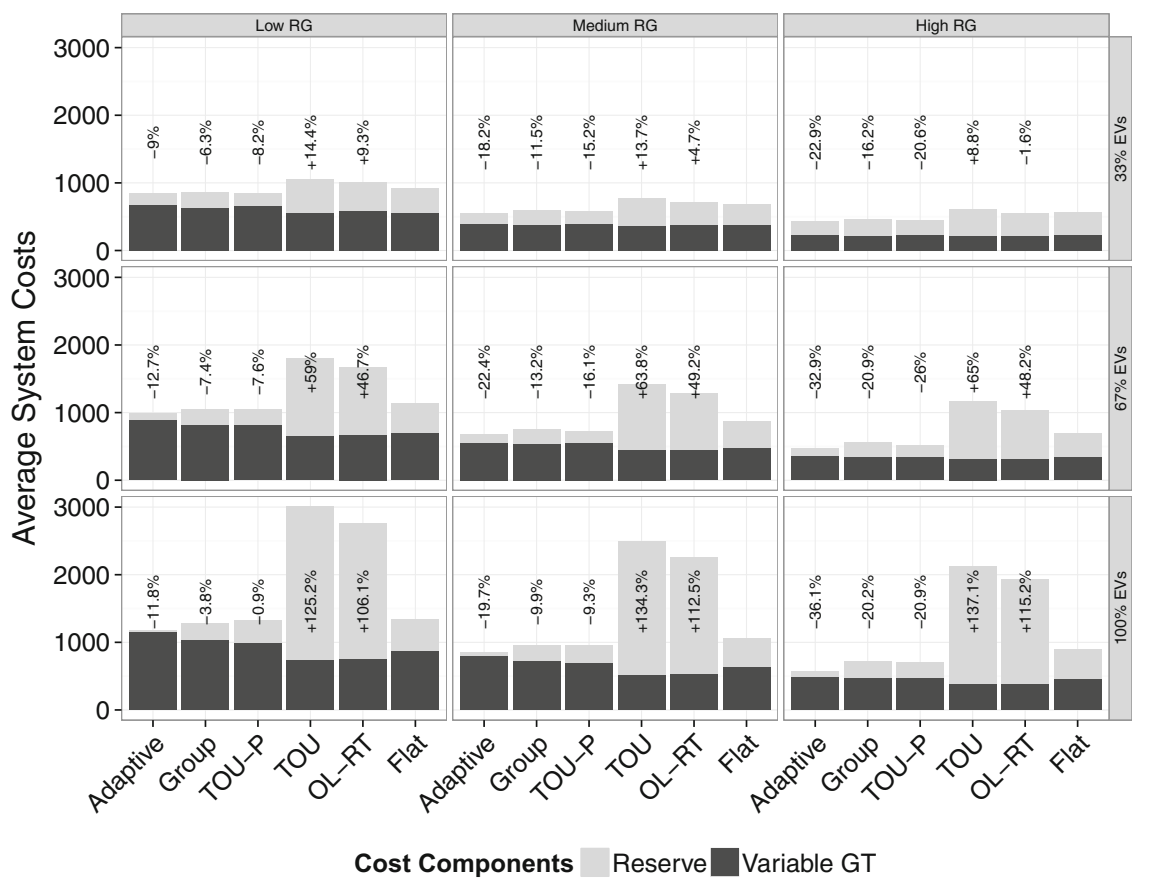

Fig. 5 System costs and cost components for different control and power system scenarios

\subsection{Aggregate system costs}

Figure 5 shows system costs and cost components for different control regimes, flexible load penetration levels, and installed renewable generation (RG) capacities. Furthermore, the percentage differential to the uncoordinated scenario (Flat) is shown. Naturally, system costs are decreasing in higher RG capacities and increasing in EV penetration level. However, the various coordination approaches handle the scenarios with very different results. Independent of the scenario, openloop real-time and time-of-use pricing system costs greatly exceed the cost under the uncoordinated regime. The described rate modifications help reduce power system costs compared to the flat pricing benchmark case. Looking in more detail at the desynchronization approaches it can be observed that power surcharges perform best in situation with limited load flexibility. Group pricing distributes charging load over more time slots and thus improves system efficiency in scenarios with a larger number of EVs. The highest savings are realized in the scenario with large wind capacity and intermediate EV penetration where TOU-P decreases system costs by $26 \%$ and randomized group pricing achieves reductions of almost $21 \%$. At the same time closed-loop adaptive pricing emerges as the most effective coordination means. Yet, the gap to the second best coordination option will largely depend on the analysis scenario at hand: In the scenarios with $33 \%$ EVs the desynchronized posted price approaches (especially TOU-P) achieve results very close to the closed 



Fig. 6 Effects of noise level and the number of groups on aggregate charging load (97.5\% quantiles), conventional generation usage and power system costs

loop approach. For higher EV penetration levels the gap widens and we see a reversal between TOU-P and group pricing cost results. In the most constrained system configuration (low RG capacity, $100 \% \mathrm{EV}$ penetration) the TOU-P savings over flat pricing collapse to $0.9 \%$ whereas group pricing achieves savings of $3.8 \%$-compared to $11.8 \%$ under adaptive pricing.

In summary, we find that the more renewable generation and flexible load are present in the system, the better more complex pricing schemes fare compared to simple ones. In the scenario with low RG and limited flexibility the flat tariff does fairly well. This resonates with recent observations that RES integration does not require system changes up to levels of $20 \%$ (IEA 2014). For intermediate system complexity desynchronized posted price approaches constitute a viable alternative. Finally, in settings with very flexible loads adaptive pricing schemes may become a necessity.

\subsection{Group design sensitivity}

Group pricing achieves promising results with respect to over-coordination and RES utilization. To better understand its application in practice, we investigate the effects of the randomization amount and the number of groups to provide design guidelines for implementing effective group tariffs. This analysis relies on the challenging scenario with high RG capacity and $100 \% \mathrm{EV}$ penetration.

The left panel of Fig. 6 depicts the $2.5 \%$ highest load values over a 12 weeks simulation period for different randomization levels and group sizes. The peak loads are decreasing in both the number of groups and the noise level applied for creating randomized electricity rates. Given greater randomization, group rates will be less homogeneous which reduces load concentration. Similarly, more groups reduce the number of vehicles reacting to a specific rate. Most of the peak load reduction potential can already be achieved with 25 groups. The center panel shows the average weekly conventional generation over the simulation period. We can observe an interdependency between the number of groups and the rate randomization level: With a single group CG usage is increasing in the rate randomization level. In this case, the random component dilutes the information on wind generation availability, resulting in the same load concentrations as under open-loop RT pricing while 
ignoring availability of wind generation. However, when moving to a larger number of groups some randomization is necessary to tap into the desynchronization potential of group pricing. For a smaller number of groups a low randomization level is optimal, otherwise the information on available wind generation will again be too diluted. For an increasing number of groups, conventional generation can only be further reduced if a stronger rate randomization (larger $\sigma$ ) is applied: Group rates become more distinct and coordination improves. Over a large number of groups the "average rate" will reflect the original generation availability signal. Combining these insights on the aggregate system cost level, the pattern exhibited by conventional generation requirements retains - system costs are minimized at an intermediate level of randomization (right panel). Also, system costs are decreasing in the number of groups with the minimum obtained under personal pricing $(900$ groups). However, the difference between 25 and 900 groups is rather limited.

\section{Conclusions and implications}

Recent research has been somewhat pessimistic concerning the potentials of pricebased coordination for demand side management due to the occurrence of load synchronization effects. Starting from regular real-time and time-of-use pricing approaches, we argue that careful modifications of these rates can reduce these load synchronization effects. This motivates the introduction of rate desynchronization approaches. First, we presented a power-based surcharge that induces more distributed charging behavior. Furthermore, we proposed individualization of residential electricity rates and present group tariffs.

Our analysis indicates that in a system with a low share of flexible loads and low renewable generation capacities flat pricing may be sufficient. Yet, increasing levels of renewable generation increases the benefits that can be tapped into by means of demand response and higher levels of flexible loads expand the number of options for system control. Standard price coordination approaches (OL-RT, TOU) result in load synchronization and lead to higher system costs than under flat pricing. The presented rate modifications can greatly reduce this over-coordination and help to reduce costs through improved integration of renewable generation. Power surcharges perform best in situations with limited load flexibility. Group pricing achieves the most promising results in scenarios with a large number of EVs. This pricing regime effectively balances between reduction of load-synchronization and incentives to exploit high generation hours. With increasing renewable generation and flexible load penetration the performance gap between closed-loop adaptive pricing and group pricing increases.

\subsection{Policy recommendations}

Reforming electricity pricing is a key task for all regulators faced with the integration of high levels of renewable generation (Kiesling 2010). While adaptive prices and local markets may achieve higher coordination efficiency than open-loop posted prices, their implementation may be prone to failure due to lack of customer 
acceptance (Woo et al. 2008; Dütschke and Paetz 2013). Hence, closed-loop adaptive pricing remains a somewhat distant vision for retail markets. In the short and medium term, regulators should strive to implement dynamic yet reliable price signals. Group pricing as outlined in this article extends today's control of storage heaters in residential households. ${ }^{11}$ Digital smart grid technologies facilitate easy and effective grouping of customers. Adapting such pricing schemes would not expose customers to price or quantity risk but energy suppliers would retain the possibility of coordinating flexible loads. Therefore, the introduction of randomness allows providers to improve utilization levels and profitability (Jerath et al. 2010). On the other hand, customers are given access to new lower cost consumption options.

\subsection{Limitations and opportunities for future research}

Naturally, the reported results are affected by the underlying assumptions. We want to discuss these limitations and suggest avenues for future research to explore extensions or relaxations of the work at hand. Price-based control can be combined with direct control options in a more comprehensive DSM portfolio design problem. Furthermore, other appliances also offer significant DSM potentials with distinct individual characteristics (for example appliances with fixed load profiles). Accounting for heterogeneous load types offers new opportunities for group composition based on customer segmentation and would increase the generality of our results. Our electric vehicle optimization assumed perfect knowledge of future driving plans on behalf of the drivers. This assumption overestimates electric vehicle flexibility (Schuller et al. 2015). Finally, participation constraints on behalf of customers may also reduce the effectiveness of the proposed coordination schemes and may result in additional (contracting) costs. This may be solved by applying mechanism design principles (Fahrioglu and Alvarado 2000) or considering non-uniform participation rates in the evaluation (Wijaya et al. 2013).

Open Access This article is distributed under the terms of the Creative Commons Attribution 4.0 International License (http://creativecommons.org/licenses/by/4.0/), which permits unrestricted use, distribution, and reproduction in any medium, provided you give appropriate credit to the original author(s) and the source, provide a link to the Creative Commons license, and indicate if changes were made.

\section{References}

Albadi, M.H., and E. El-Saadany. 2008. A summary of demand response in electricity markets. Electric Power Systems Research 78(11): 1989-1996.

Bitar, E., Low, S., 2012. Deadline differentiated pricing of deferrable electric power service. In IEEE conference on decision and control, 4991-4997.

Blumsack, S., and A. Fernandez. 2012. Ready or not, here comes the smart grid!. Energy 37(1): 61-68. Bohn, R. E., 1982. Spot pricing of public utility services. Ph.D. thesis, Massachusetts Institute of Technology, Energy Laboratory.

\footnotetext{
11 Grid operators determine groups of storage appliances and transmit intervals on group level to desynchronize these large loads (Hastings 1980).
} 
Borenstein, S., M. Jaske, and A. Rosenfeld. 2002. Dynamic pricing, advanced metering, and demand response in electricity markets. Technical report, Center for the Study of Energy Markets, Berkley

Callaway, D.S., and I.A. Hiskens. 2011. Achieving controllability of electric loads. Proceedings of the IEEE 99(1): 184-199.

Dütschke, E., and A.-G. Paetz. 2013. Dynamic electricity pricing-which programs do consumers prefer? Energy Policy 59: 226-234.

Fahrioglu, M., and F.L. Alvarado. 2000. Designing incentive compatible contracts for effective demand management. IEEE Transactions on Power Systems 15(4): 1255-1260.

Faruqui, A., D. Harris, and R. Hledik. 2010. Unlocking the $€ 53$ billion savings from smart meters in the eu: how increasing the adoption of dynamic tariffs could make or break the eu's smart grid investment. Energy Policy 38(10): 6222-6231.

Faruqui, A., and J. Palmer. 2012. The discovery of price responsiveness-a survey of experiments involving dynamic pricing of electricity. EDI Quarterly 4(1): 15-18.

Fay, S. 2008. Selling an opaque product through an intermediary: the case of disguising one's product. Journal of Retailing 84(1): 59-75.

Flath, C.M., 2013. An optimization approach for the design of time-of-use rates. In Proceedings of the 39th annual conference of the IEEE Industrial Electronics Society (IECON), Vienna.

Flath, C.M., J.P. Ilg, S. Gottwalt, H. Schmeck, and C. Weinhardt. 2014. Improving electric vehicle charging coordination through area pricing. Transportation Science 48(4): 619-634.

Franke, T., I. Neumann, F. Bühler, P. Cocron, and J.F. Krems. 2012. Experiencing range in an electric vehicle: understanding psychological barriers. Applied Psychology 61(3): 368-391.

Gan, L., U. Topcu, and S.H. Low. 2013. Optimal decentralized protocol for electric vehicle charging. IEEE Transactions on Power Systems 28(2): 940-951.

Goett, A.A., K. Hudson, and K.E. Train. 2000. Customers' choice among retail energy suppliers: the willingness-to-pay for service attributes. The Energy Journal 21(4): 1-28.

Gong, Q., S. Midlam-Mohler, V. Marano, and G. Rizzoni. 2012. Study of PEV charging on residential distribution transformer life. IEEE Transactions on Smart Grid 3(1): 404-412.

Gottwalt, S., W. Ketter, C. Block, J. Collins, and C. Weinhardt. 2011. Demand side management-a simulation of household behavior under variable prices. Energy Policy 39(12): 8163-8174.

Grünewald, P., E. McKenna, and M. Thomson. 2014. Keep it simple: time-of-use tariffs in high-wind scenarios. Renewable Power Generation IET 9(2): 176-183.

Hastings, B. 1980. Ten years of operating experience with a remote controlled water heater load management system at Detroit Edison. IEEE Transactions on Power Apparatus and Systems PAS 99(4): 1437-1441.

Homburg, C., D. Totzek, and M. Krämer. 2014. How price complexity takes its toll: the neglected role of a simplicity bias and fairness in price evaluations. Journal of Business Research 67(6): 1114-1122.

IEA. 2014. The power of transformation: Wind, sun and the economics of flexible power systems. Technical report, International Energy Agency

Jerath, K., S. Netessine, and S.K. Veeraraghavan. 2010. Revenue management with strategic customers: last-minute selling and opaque selling. Management Science 56(3): 430-448.

Jiang, Y. 2007. Price discrimination with opaque products. Journal of Revenue and Pricing Management 6(2): 118-134.

Kiesling, L.L. 2010. Promoting innovation in the electricity industry. Economic Affairs 30(2): 6-12.

Kishore, S., Snyder, L.V., 2010. Control mechanisms for residential electricity demand in smartgrids. In IEEE international conference on smart grid communications, 443-448.

Lamparter, S., Becher, S., Fischer, J.-G., 2010. An agent-based market platform for smart grids. In Proceedings of the 9th international conference on autonomous agents and multiagent systems: industry track, 1689-1696. International Foundation for Autonomous Agents and Multiagent Systems.

Lopes, J.P., S.A. Polenz, C. Moreira, and R. Cherkaoui. 2010. Identification of control and management strategies for LV unbalanced microgrids with plugged-in electric vehicles. Electric Power Systems Research 80(8): 898-906.

Los, H., de Jong, C., van Dijken, H., 2009. Realistic power plant valuations. World Power, 48-53. London: Isherwood Production.

Mohsenian-Rad, A., V.W. Wong, J. Jatskevich, R. Schober, and A. Leon-Garcia. 2010. Autonomous demand-side management based on game-theoretic energy consumption scheduling for the future smart grid. IEEE Transactions on Smart Grid 1(3): 320-331. 
Muratori, M., and G. Rizzoni. 2015. Residential demand response: dynamic energy management and time-varying electricity pricing. IEEE Transactions on Power Systems PP(99): 1-10.

Petersen, M., Edlund, K., Hansen, L., Bendtsen, J., Stoustrup, J., 2013. A taxonomy for modeling flexibility and a computationally efficient algorithm for dispatch in smart grids. In American control conference, 1150-1156.

Qian, K., C. Zhou, M. Allan, and Y. Yuan. 2011. Modeling of load demand due to EV battery charging in distribution systems. IEEE Transactions on Power Systems 26(2): 802-810.

Ramchurn, S.D., Vytelingum, P., Rogers, A., Jennings, N., 2011. Agent-based control for decentralised demand side management in the smart grid. In 10th international conference on autonomous agents and multiagent systems.

Ramchurn, S.D., P. Vytelingum, A. Rogers, and N.R. Jennings. 2012. Putting the 'Smarts' into the smart grid: a grand challenge for artificial intelligence. Communications of the ACM 55(4): 86-97.

Schuller, A., B. Dietz, C. Flath, and C. Weinhardt. 2014. Charging strategies for battery electric vehicles: economic benchmark and V2G potential. IEEE Transactions on Power Systems 29(5): 1-9.

Schuller, A., C.M. Flath, and S. Gottwalt. 2015. Quantifying load flexibility of electric vehicles for renewable energy integration. Applied Energy 151: 335-344.

Schweppe, F.C., M.C. Caramanis, R.D. Tabors, and R.E. Bohn. 1988. Spot pricing of electricity. Boston: Kluwer Academic Publishers.

Shinwari, M., A. Youssef, and W. Hamouda. 2012. A water-filling based scheduling algorithm for the smart grid. IEEE Transactions on Smart Grid 3(2): 710-719.

Sioshansi, R. 2012. Modeling the impacts of electricity tariffs on plug-in hybrid electric vehicle charging, costs, and emissions. Operations Research 60(3): 506-516.

Sioshansi, R., R. Fagiani, and V. Marano. 2010. Cost and emissions impacts of plug-in hybrid vehicles on the Ohio power system. Energy Policy 38(11): 6703-6712.

Strbac, G. 2008. Demand side management: benefits and challenges. Energy Policy 36(12): 4419-4426.

Subramanian, A., M.J. Garcia, D.S. Callaway, K. Poolla, and P. Varaiya. 2013. Real-time scheduling of distributed resources. IEEE Transactions on Smart Grid 4(4): 2122-2130.

Van Den Briel, M., Scott, P., Thiébaux, S., 2013. Randomized load control: A simple distributed approach for scheduling smart appliances. In Proceedings of the twenty-third international joint conference on artificial intelligence, 2915-2922. AAAI Press.

van Dijken, H., van Abbema, D., Los, H.S., de Jong, C., 2010. The value of starting up the power plant. World Power, 48-53. London: Isherwood Production.

Vandael, S., N. Boucké, T. Holvoet, K. De Craemer, and G. Deconinck. 2011. Decentralized coordination of plug-in hybrid vehicles for imbalance reduction in a smart grid. In The 10th international conference on autonomous agents and multiagent systems, Richland, SC, 803-810.

Varaiya, P.P., F.F. Wu, and J.W. Bialek. 2011. Smart operation of smart grid: risk-limiting dispatch. Proceedings of the IEEE 99(1): 40-57.

Wijaya, T.K., Papaioannou, T.G., Liu, X., Aberer, K., 2013. Effective consumption scheduling for demand-side management in the smart grid using non-uniform participation rate. In Sustainable internet and ICT for sustainability (SustainIT), 1-8. doi:10.1109/SustainIT.2013.6685188

Woo, C., E. Kollman, R. Orans, S. Price, and B. Horii. 2008. Now that california has AMI, what can the state do with it? Energy Policy 36(4): 1366-1374.

Wunderlich, P., J. Kranz, D. Totzek, D. Veit, and A. Picot. 2013a. The impact of endogenous motivations on adoption of it-enabled services the case of transformative services in the energy sector. Journal of Service Research 16(3): 356-371.

Wunderlich, P., Kranz, J., Veit, D., 2013b. Beyond carrot-and-stick: How values and endogenous motivations affect residential green IS adoption. In Proceedings of the international conference on information systems. 